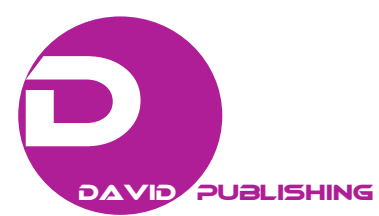

\title{
Role of Perfusion CT Parameter Measurements in Differentiating Treatment Induced Necrosis from Recurrent Tumor
}

\author{
Asim Shaukat, Nosheen Ahmad and Aamir Shaukat \\ Department of Radiology and Medical Imaging, Punjab Medical College, Allied Hospital, Faisalabad, Punjab 38000, Pakistan
}

\begin{abstract}
OBJECTIVES: To assess the use of perfusion CT parameters in differentiating treatment-induced necrosis from recurrent or progressive lesions after radiotherapy for brain tumor. DESIGN: Cross-sectional study. SETTING: Department of Radiology Allied Hospital, Faisalabad. PERIOD: From January 2013 to June 2014. PATIENTS AND METHOD: Forty-five consecutive patients who presented with recurrent/progressive enhancing brain lesion on imaging studies after radiotherapy for brain tumor were included in the study, 28 males $(62.2 \%)$ and 17 females (37.8\%). Their age ranged from 40 to 65 years, mean age $52.22 \pm 7.8$ years, referred to the radiology department for follow up imaging from neuro-oncology department of Allied hospital Faisalabad. All patients had an initial non-contrast CT brain, then Perfusion CT (PCT) followed by histopathological examination. The obtained perfusion CT images were used for image processing. Permeability surface area product (PS), cerebral blood volume (CBV), cerebral blood flow (CBF) and mean transit time (MTT) were calculated from the enhancing lesion as well as from normal healthy cortex and the results were compared to histopathological examination results. RESULTS: Of 45 patients, 14 were diagnosed with treatment-induced necrosis (TIN) and 31 had recurrent or progressive tumor (RPT). Patients with treatment-induced necrosis showed significantly lower mean PS values than those with recurrent or progressive tumor $(1.8 \pm 0.28$ versus $3.12 \pm 0.34 \mathrm{~mL} / 100 \mathrm{~g} / \mathrm{min})$. Patients with treatment-induced necrosis also showed lower CBV $(1.41 \pm 0.22 \mathrm{~mL}$ versus $1.63 \pm 0.17 \mathrm{~mL})$, lower $\mathrm{CBF}(1.39 \pm 0.31 \mathrm{~mL} / \mathrm{min}$ versus $1.87 \pm 0.67 \mathrm{~mL} / \mathrm{min})$, and higher MTT $(1.84 \pm 0.54 \mathrm{~s}$ versus $1.37 \pm 0.55 \mathrm{~s})$ than those with recurrent or progressive tumor group. CONCLUSIONS: PCT parameters can be used in differentiating treatment-induced necrosis from recurrent or progressive disease in patients with previously treated brain tumor with radiotherapy. Among PCT parameters, permeability measurements (PS) has more sensitivity and accuracy in differentiating these two groups.
\end{abstract}

Key words: Perfusion CT imaging (PCT), treatment induced necrosis (TIN), recurrent or progressive tumor (RPT), permeability surface (PS), cerebral blood volume (CBV).

\section{Introduction}

Differentiating treatment-induced necrosis from recurrent tumor is a great challenge in neuro-oncology. These two entities after brain tumor treatment are very similar on routine follow-up imaging. Distinguishing radiation induced necrosis from tumor recurrence is essential for diagnosis because the treatment strategies for tumor recurrence and radiation necrosis are different.

Conventional MR imaging features and MR spectroscopic imaging have been used to differentiate

Corresponding author: Asim Shaukat, MBBS, FCPS, associate professor, research field: radiology. radiation necrosis from recurrent brain tumors but with mixed success [1].

MRI is routinely used in the follow up and monitoring of patients with brain tumor that provides anatomical information; however, it suffers from limitations in differentiating treatment necrosis from tumor recurrence.

The Perfusion-CT technique has been recently studied to investigate the role of perfusion maps (tumor blood volume, blood flow and vascular permeability) for evaluating brain neoplasms, because of the growing interest in the non-invasive way to assess the tumor vascularity [2]. 
Perfusion computed tomography (PCT) allows the quantitative assessment of hemodynamic characteristics of brain tissue, tumor neoangiogenesis and neovascularization that play an important role in tumor growth and spread, enables the prediction of tumor grade and the assessment of treatment response and prognosis [3].

Permeability surface (PS) represents a different aspect of neoangiogenesis and neovascularity that provides additional physiologic information about recurrent/progressive enhancing lesions [4]. Tumor blood vessels have defective and leaky endothelium. Permeability surface (PS) characterizes the diffusion of some of the contrast agent from the abnormal tumor blood vessels into the interstitial space. Thus, in vivo measurement of tumor vessel permeability is important for grading tumors, to access the response of tumors to antiangiogenic therapy and to differentiate tumor recurrence from radiation necrosis.

The perfusion and blood volume are the more accurate maps, however, vascular permeability as suggested by Miles et al. [5] is the most sensitive to tissue changes from normal to malignant state.

The purpose of our study was to evaluate a non-invasive way of quantifying and classifying the perfusion parameters by using PCT in the differentiation of recurrent intra-axial brain tumor and treatment induced necrosis who presented with a recurrent/progressive enhancing lesion on follow up imaging studies.

\section{Material and Methods}

We approached 45 patients from neuro-oncology department of Allied Hospital Faisalabad who ranged from 45-70 years of age (mean $45.13 \pm 5.54$ ) of both genders.

All patients have pretreatment diagnosis of grade 4 glial tumor, glioblastoma multiforme (GBM). This study was undertaken in Department of Radiology, Allied Hospital Faisalabad and was carried out over a period of 18 months from January 2013 to June 2014.
All patients showing intraaxial enhancing brain lesions on imaging studies with previous history of brain tumor radiotherapy three months back were recruited in our study. The included patients had no known biopsy results at the time of examination. Exclusion criteria for PCT were poor renal function and patient motion or instability.

All the patients were examined by 128 slice GE CT scanner and included a Non Contrast CT scan NCCT and PCT. PCT was then followed by biopsy in order to make a histopathological diagnosis of enhancing lesions on CT scan.

A low dose unenhanced CT brain was performed before obtaining the perfusion scan in order to locate the lesion and to plan the slice positions of PCT; the acquisition parameters were $80 \mathrm{kVp}$ and $120 \mathrm{mAs}$. Dynamic perfusion CT was performed with $4 \mathrm{~cm}$ plane coverage (8 slices of $5 \mathrm{~mm}$ thickness). PCT imaging was acquired as a cine series of 50 seconds, beginning 5 seconds after injection of $50 \mathrm{~mL}$ non-ionic iodine contrast using a power injector.

Commercially available software (perfusion CT; GE optima 660) was used to calculate parametric maps of erebral blood volume (CBV), cerebral blood flow $(\mathrm{CBF})$, permeability surface (PS) and mean transit time (MTT) by using baseline CT perfusion data. Arterial input and venous output time-attenuation curves were generated, with regions of interest manually drawn by the CT technologist performing the study, mostly in the artery with the greatest peak and slope on time-attenuation curves, and the superior sagittal sinus, respectively.

PCT was assessed by a radiologist for permeability surface (PS), cerebral blood volume (CBV), cerebral blood flow (CBF), mean transit time (MTT). Values were obtained from the lesion as well as from the normal cortex.

Data was analyzed on SPSS version 17 and was expressed as mean \pm SD for each perfusion parameter. For all patients, histopathological results were considered the reference standard. 


\section{Results}

Among 45 patients, 14 were diagnosed with treatment-induced necrosis and 31 had recurrent or progressive tumor (Table 1).

Patients with treatment induced necrosis showed significantly lower mean PS, CBV and CBF values and higher MTT values than those with recurrent or progressive tumor. Among these parameters, mean PS and $\mathrm{CBV}$ remained significantly associated with differentiating the two groups $(P=0.0001$ and 0.001 , respectively) (Table 2 ).

Frequency of true positive, false positive, true negative, false negative patients with PS and CBV pathology can be found in Tables 3 and 4.

Sensitivity, specificity, positive predictive value (PPV), negative predictive value (NPV) and accuracy are presented in Table 5. Using previously described cut-off value of 2.5 and 1.5 for PS and CBV,

Table 1 Patient diagnosis.

\begin{tabular}{lll}
\hline Diagnosis & Frequency & Percentage (\%) \\
\hline Treatment induced necrosis & 14 & 31.1 \\
Recurrent or progressive tumor & 31 & 68.9 \\
Total & 45 & 100 \\
\hline
\end{tabular}

Table $2 P$ value, mean and SD value of perfusion parameters for recurrent tumor and radiation induced necrosis.

\begin{tabular}{lllllll}
\hline \multirow{2}{*}{ Perfusion parameter } & \multicolumn{2}{c}{ Treatment induced necrosis (14 patients) } & \multicolumn{2}{c}{ Recurrent or progressive tumor (31 patients) } \\
\cline { 2 - 3 } & Mean & Standard deviation & & Mean & Standard deviation & \\
\hline PS $(\mathrm{mL} / 100 \mathrm{~g} / \mathrm{min})$ & 1.8 & 0.28 & 3.12 & 0.34 & 0.0001 \\
$\mathrm{CBV}(\mathrm{mL} / 100 \mathrm{~g})$ & 1.41 & 0.22 & 1.63 & 0.17 & 0.001 \\
$\mathrm{CBF}(\mathrm{mL} / 100 \mathrm{~g} / \mathrm{min})$ & 1.39 & 0.31 & 1.87 & 0.67 & 0.002 \\
MTT $(\mathrm{sec})$ & 1.84 & 0.54 & 1.37 & 0.55 & 0.011 \\
\hline
\end{tabular}

Table 3 PS-pathology correlation.

\begin{tabular}{lll}
\hline & Frequency & Percentage (\%) \\
\hline True positive & 29 & 64.45 \\
False positive & 1 & 2.22 \\
True negative & 13 & 28.89 \\
False negative & 2 & 4.44 \\
Total & 45 & 100 \\
\hline
\end{tabular}

Table 4 CBV-pathology correlation.

\begin{tabular}{lll}
\hline & Frequency & Percentage (\%) \\
\hline True positive & 27 & 60 \\
False positive & 2 & 4.44 \\
True negative & 12 & 26.67 \\
False negative & 4 & 8.89 \\
Total & 45 & 100 \\
\hline
\end{tabular}

Table 5 Comparison of sensitivity, specificity, accuracy of PS and CBV in differentiating TIN and RPT.

\begin{tabular}{lll}
\hline & PS & CBV \\
\hline Cutoff value & 2.5 & 1.5 \\
Sensitivity (\%) & 93.5 & 81.7 \\
Specificity (\%) & 92.86 & 85.7 \\
PPV (\%) & 96.67 & 93.1 \\
NPV (\%) & 86.67 & 75 \\
Diagnostic Accuracy & 93.3 & 86.67 \\
\hline
\end{tabular}




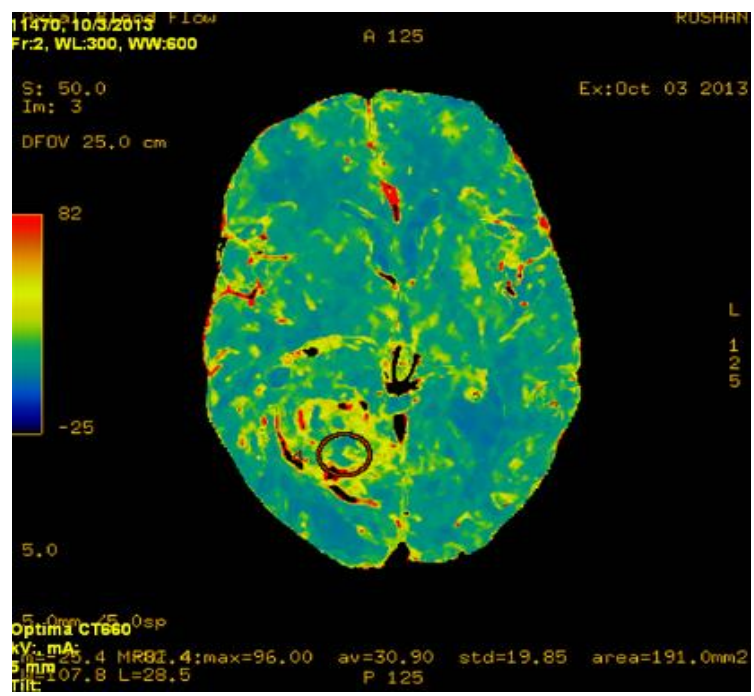

(a) $\mathrm{CBF}$

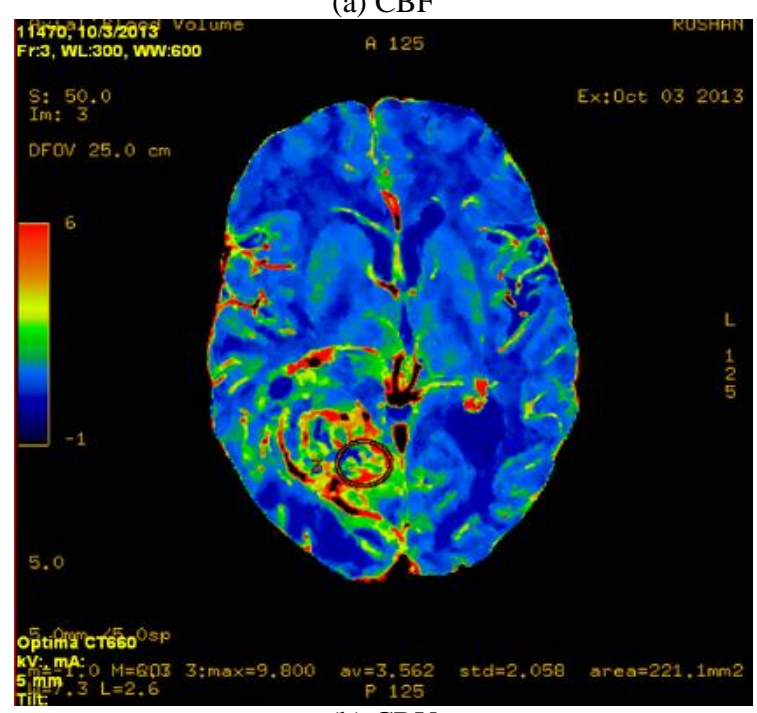

(b) $\mathrm{CBV}$

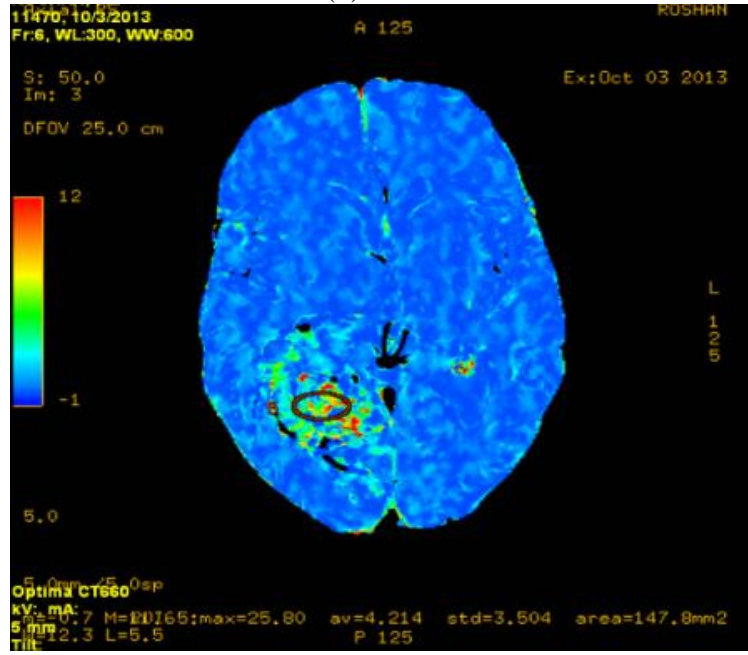

(c) PS

Fig. 1 PCT images demonstrate high CBF (a), CBV(b) and PS (c) values suggesting recurrent right occipitoparietal tumor.
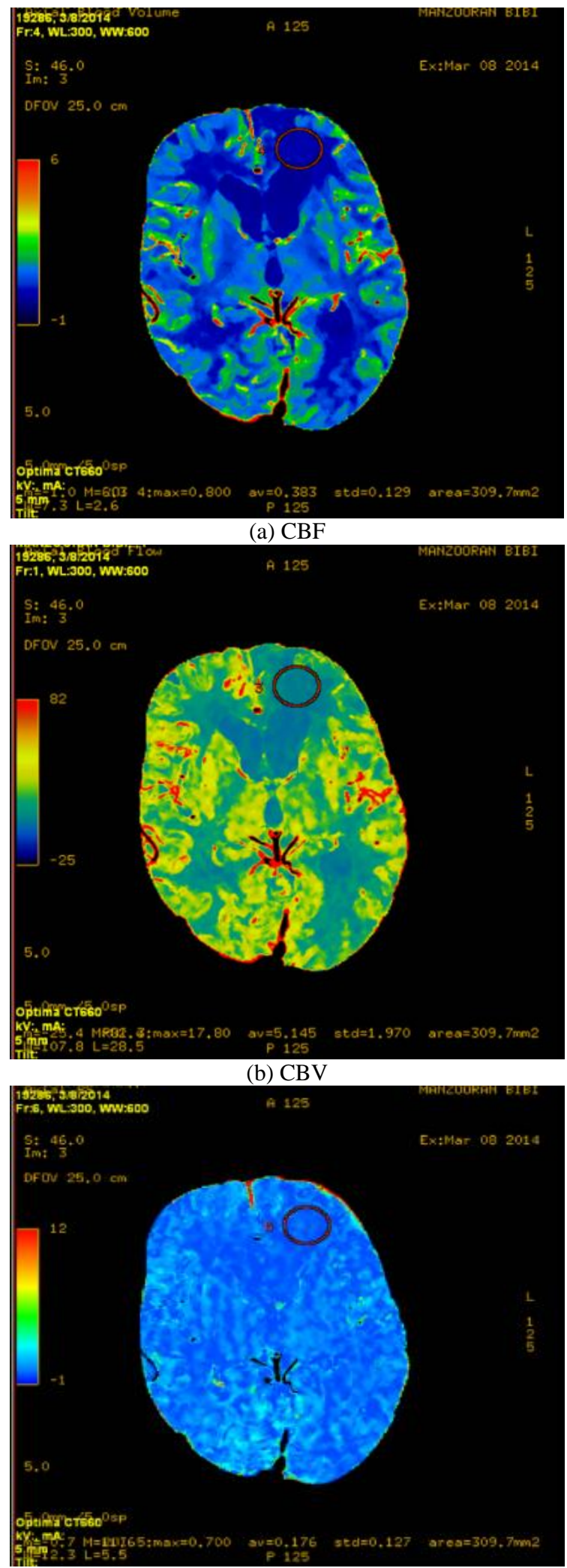

(c) PS

Fig. 2 Perfusion CT images showing low CBF (a), CBV(b) and PS (c) values in radiation inducednecrosis in left frontal region. 
respectively [4], there was higher sensitivity, specificity, PPV, NPV and accuracy with the PS parameter.

\section{Discussion}

The current gold standard for brain tumor grading is biopsy and histopathologic assessment. This technique, however, is invasive and has own limitations including sampling error, interobserver variation and tumor heterogeneity [6].

Recently, there is significant interest in developing noninvasive methods that determine whether a contrast-enhancing lesion is due to treatment necrosis or tumor recurrence. Several imaging techniques have been evaluated for this purpose. Traditionally, perfusion imaging of brain tumors has been performed with MRI, but recently, perfusion $\mathrm{CT}$ has been investigated to achieve this goal.

The perfusion CT can be used for preoperative grading of the gliomas, in particularly for the differentiation of low from high-grade astrocytomas because this technique provides complementary information about tumor hemodynamics, not available with conventional CT or MR. The potential role of PCT lies in the differential diagnosis between radiation necrosis and recurrence in patients who have undergone radiotherapy, in the evaluation of the response to the anti-angiogenetic therapy, and its ability to detect the biological effects to treatment by depicting early microvascularization changes before tumor size modifications.

Perfusion CT is able to differentiate tumor recurrence from treatment necrosis with reasonableaccuracy $[3,7,8]$. Potential advantages of PCT over MR perfusion include faster scanning times, measurement of absolute hemodynamic perfusion parameters, relatively easy post processing, lower cost, and lack of susceptibility artifacts $[3,8]$.

PCT is also advantageous over perfusion weighted imaging (PWI) in giving the linear relationship between density changes and the contrast agent concentration, lack of sensitivity to flow and the high spatial resolution. Moreover, susceptibility artefacts generated by hemorrhage or various mineral depositions with PWI, can complicate the perfusion analysis in post-treatment tumor patients [9]. Absolute measurement of the perfusion parameters is provided by PCT, whereas MR imaging estimates are mostly relative to the normal brain parenchyma.

Perfusion MRI is capable of quantifying microvessel density (vascularity) and permeability of brain tissue by assessment of the relative cerebral blood volume (rCBV) and has been used for grading, histological differentiation and assessment of prognosis in glioma patients. Hoefnagels et al. showed a sensitivity and specificity of $70.0 \%$ and $92.9 \%$, respectively and positive (PPV) and negative predictive values (NPV) of $93.3 \%$ and $68.4 \%$, respectively in predicting the tumor recurrence by logistic regression analysis of rCBV map [10].

Radiation necrosis is indistinguishable from recurrent GBM at conventional contrast-enhanced MR imaging on the basis of lesion characteristics [11]. Single voxel MRS has sensitivity of $50 \%$ in distinguishing these two entities according to Chernov et al. [1].

Malignant brain tumors are characterized by neovascularity and increased angiogenesis. Perfusion imaging has been proposed as a method to assess tumor grade, tumor revolution, and treatment response. Increased vascular permeability has also been correlated with malignancy and has been evolving as a surrogate marker of tumor angiogenesis and, thus, tumor grade [12].

Kumar et al. [13] described the features that favor radiation necrosis over recurrent and/or progressive tumor on imaging: conversion from no enhancement to an enhancing lesion, remote new enhancement and soap-bubble or Swiss cheese pattern of enhancement.

Jain et al. [7] described that recurrent tumors showed higher $\mathrm{CBV}$ and $\mathrm{CBF}$, and lower MTT. For nCBV, a 
cutoff point of 1.65 was found to have a sensitivity of $83.3 \%$ and a specificity of $100 \%$ to diagnose recurrent tumor versus radiation necrosis. Similarly, cut-off points of 1.28 and 1.44 resulted in sensitivity and specificity values of $94.4 \%$ and $87.5 \%$ for $\mathrm{nCBF}$ and $94.4 \%$ and $75 \%$ for MTT respectively. Mitsuyak et al. reported a cut off value of nCBV ratio of greater than 3.0 which has the sensitivity and specificity of $81.3 \%$ and $100 \%$ respectively for identifying recurrent tumors from radiation necrosis [14].

Radiation necrosis shows lower PS value than recurrent tumor $(1.8 \pm 0.8$ versus $3.6 \pm 1.6 \mathrm{~mL} / 100$ $\mathrm{g} / \mathrm{min} ; \quad P$ value $=0.001)$. Additionally, radiation necrosis shows higher rMTT $(1.4 \pm 0.4$ versus $1.0 \pm 0.4$; $P$ value $=0.018)$, lower $\mathrm{rCBF}(1.2 \pm 0.5$ versus $2.6 \pm$ 1.7), and lower CBV $(1.2 \pm 0.3$ versus $2.1 \pm 0.7)$ compared with recurrent tumors, probably due to the fact that recurrent tumors have leakier blood vessels compared with treatment induced necrosis [4]. PS and CBV have higher sensitivity and specificity to differentiate these two entities [4].

Our study concluded that treatment induced necrosis shows lower PS, CBV and CBF values and higher MTT values compared with recurrent tumors which is similar to prior studies [4]. High sensitivity and specificity were obtained by using a cut off value of CBV 1.5 (sensitivity, $81.7 \%$; specificity, $85.7 \%$ ) and of PS 2.5 (sensitivity, 93.5\%; specificity, 92.86\%) to differentiate the two groups [15]. Together, these findings are similar to previous studies $[4,15]$. The diagnostic accuracy was higher for PS compared with CBV.

PCT has the potential to be a useful tool for brain tumor assessment and is relatively more sensitive than MRI techniques $[1,10,11]$ as also depicted in our study and is capable of providing a categorical diagnosis of true tumor versus enhancement change due to treatment-induced necrosis.

Potential limitation of our study was small sample size, restricted anatomical coverage, radiation exposure compared with MR perfusion techniques.

\section{Conclusion}

Perfusion CT imaging shows promising results for distinguishing brain tumor recurrence from radionecrosis. PCT parameters can be used in patients with previously treated brain tumors to differentiate treatment induced necrosis from recurrent/progressive tumor so to avoid the invasive biopsy procedures.

\section{References}

[1] Chernov, M., Hayashi, M., Izawa, M., Ochiai, T., Usukura, M., Abe, K., Ono, Y., Muragaki, Y., Kubo, O., Hori, T., and Takakura, K. 2005. "Differentiation of the Radiation-Induced Necrosis and Tumor Recurrence after Gamma Knife Radiosurgery for Brain Metastases: Importance of Multivoxel Proton MRS." Minimally Invasive Neurosurgery 48 (4): 228-34.

[2] Miles, K. A., and Griffiths, M. R. 2003. "Perfusion CT: A Worthwhile Enhancement?" British Journal of Radiology 76 (904): 220-31.

[3] Jain, J., Ellika, S. K., Scarpace, L., Schultz, L. R., Gutierrez, J., Patel, S. C., Ewing, J., and Mikkelsen, T. 2008. "Quantitative Estimation of Permeability Surface-Area Product in Astroglial Brain Tumors Using Perfusion CT and Correlation with Histopathologic Grade." AJNR American Journal of Neuroradiology 29 (4): 694-700.

[4] Jain, R., Narang, J., Schultz, L., Scarpace, L., Saksena, S., Brown, S., Rock, J. P., Rosenblum, M., Gutierrez, J., and Mikkelsen, T. 2011. "Permeability Estimates in Histopathology Proven Treatment Induced Necrosis Using Perfusion CT Scan These Add to Other Perfusion Parameters in Differentiating From Recuurent/Progressive Tumors?" AJNR American Journal of Neuradiology 32 (4): 658-63.

[5] Miles, K. A. 2003. "Perfusion CT for the Assessment of Tumor Vascularity: Which Protocol?" British Journal of Radiology 76 (1): 536-42.

[6] Law, M., Young R. J., Babb, J. S., Peccerelli, N., Chheang, S., Gruber, M. L., Miller, D. C., Golfinos, J. G., Zagzag, D., and Johnson, G. 2008. "Gliomas: Predicting Time to Progression or Survival with Cerebral Blood Volume Measurement at Dynamic Susceptibility-Weighted Contrast-Enhanced Perfusion MR Imaging." Radiology 247 (2): 490-8.

[7] Jain, R., Scarpace, L., Ellika, S., Schultz, L. R., Rock, J. P., Rosenlum, M. L., Patel, S. C., Lee, T. Y., and Mikkelsen, T. 2007. "First-Pass Perfusion Computed Tomography: Initial Experience in Differentiating Recurrent Brain Tumors from Radiation Effects and Radiation Necrosis." 


\section{Treatment Induced Necrosis from Recurrent Tumor}

Neurosurgery 61 (4): 778-86.

[8] Ellika, S. K., Jain, R., Patel, S. C., Scarpace, L., Schultz, L. R., Rock, J. P., and Mikkelsen, T. 2007. "Role of Perfusion CT in Glioma Grading and Comparison with Conventional MR Imaging Features." AJNR American Journal of Neuroradiology 28 (6): 1981-87.

[9] Schramm, P. 2007. "High Concentration Contrast Media in Neurological Multidetector-Row CT-Applications: Implications for Improved Patient Management in Neurology and Neurosurgery." Neurordiology 49 (Suppl 1): S35-45.

[10] Hoefnagels, F. W. A., Lagerwaard, F. J., Sanchez, E., Haasbeek, C. J. A., Knol, D. L., Slotman, B. J., and Vandertop W. P. 2009. "Radiological Progession of Cerebral Metastases after Radiosurgery: Assessment of Perfusion MRI for Differentiating Between Necrosis and Recurrence." Journal of Neurology 256 (6): 878-97.

[11] Barajas, R. F., Chang, J. S., Segal, M. R., Parsa, A. T., McDemott, M. W., Berger, M. S., and Cha, S. 2009. "Differentiation of Recurrent Glioblastoma Multiforme from Radiation Necrosis after External Beam Radiation Therapy with Dynamic Susceptibility-Weighted Contrast-Enhanced Perfusion MR Imaging." Radiology
253 (2): 486-96.

[12] Dijke, C. F., Brasch, R. C., Roberts, T. P., Weidner, N., Mathur, A., Shames, D. M., Mann J. S., Demsar, F., Lang, P., and Schwicket, H. C. 1996. "Mammary Carcinoma Model: Correlation of Macromolecular Contrast-Enhanced MR Imaging Characterization of Tumor Microvasclature and Histologic Capillary Density." Radiology 2000 (2): 377-84.

[13] Kumar, A. J., Leeds, N. E., Fuller, G. N., Van Tassel, P., Maor, M. H., Sawaya, R. E., and Levin, V. A. 2000. "Malignant Gliomas: MR Imaging Spectrum of Radiation Therapy- and Chemotherapy-Induced Necrosis of the Brain after Treatment." Radiology 217 (2): 377-84.

[14] Mitsuya, K., Nakasu, Y., Hayashi, N., Harada H., Nishimura, T., Ito, I., Urikura, A., Nakaya, Y., and Endo, M. 2014. "Differential Diagnosis of Tumor Recurrence and Radiation Necrosis after Radiosurgery for Brain Metastases with 320-Row Area Detector CT Perfusion Imaging." Neuro. Oncol. 165 (Suppl 2): pii83.

[15] Yeung, T. P. C., Bauman, G., Yartsev, S., Fainardi, E., Macdonald, D., and Lee, T. Y. 2015. "Dynamic Perfusion CT in Brain Tumors." European Journal of Radiology Feb 23. Pii: SO720-048X(15)00080-7. 